ARTICLE

Received 7 Jul 2015 | Accepted 31 May 2016 | Published 29 Jun 2016

DOI: $10.1038 /$ ncomms12112

OPEN

\title{
Finite particle size drives defect-mediated domain structures in strongly confined colloidal liquid crystals
}

\author{
Ioana C. Gârlea ${ }^{1, \dagger}$, Pieter Mulder ${ }^{1}$, José Alvarado ${ }^{1, \dagger}$, Oliver Dammone ${ }^{2}$, Dirk G.A.L. Aarts ${ }^{2}$, M. Pavlik Lettinga ${ }^{3}$,
} Gijsje H. Koenderink ${ }^{1} \&$ Bela M. Mulder ${ }^{1}$

When liquid crystals are confined to finite volumes, the competition between the surface anchoring imposed by the boundaries and the intrinsic orientational symmetry-breaking of these materials gives rise to a host of intriguing phenomena involving topological defect structures. For synthetic molecular mesogens, like the ones used in liquid-crystal displays, these defect structures are independent of the size of the molecules and well described by continuum theories. In contrast, colloidal systems such as carbon nanotubes and biopolymers have micron-sized lengths, so continuum descriptions are expected to break down under strong confinement conditions. Here, we show, by a combination of computer simulations and experiments with virus particles in tailor-made disk- and annulus-shaped microchambers, that strong confinement of colloidal liquid crystals leads to novel defect-stabilized symmetrical domain structures. These finite-size effects point to a potential for designing optically active microstructures, exploiting the as yet unexplored regime of highly confined liquid crystals.

\footnotetext{
${ }^{1}$ Department of Systems Biophysics, FOM Institute AMOLF, Science Park 104, 1098XG Amsterdam, The Netherlands. ${ }^{2}$ Department of Chemistry, Physical and Theoretical Chemistry Laboratory, University of Oxford, South Parks Road, Oxford OX1 3QZ, UK. ${ }^{3}$ Institute of Complex Systems (ICS-3),

Forschungszentrum Jülich, 52425 Jülich, Germany. †Present address: Department of Mechanical Engineering, Massachusetts Institute of Technology, 77 Massachusetts Avenue, Cambridge, Massachusetts 02139-4307, USA (J.A.). Correspondence and requests for materials should be addressed to B.M.M. (email: mulder@amolf.nl).
} 
T he study of liquid crystals in confinement has a history stretching back to the days of the development of the first twisted nematic display cells ${ }^{1}$. Confined liquid crystals attracted the attention of fundamental scientists, as they provided a window on a host of intriguing phenomena.

First and foremost, this concerned the study of topological defects, which in these systems arise both spontaneously or can be induced and modulated through the geometry of confinement and the imposed boundary conditions. Starting in the 1970s a major effort was undertaken to understand and classify these defect structures ${ }^{2-5}$. In the 1980 s the focus was on spherical confinement geometry ${ }^{5,6}$, spurred in part by the development of polymer dispersed liquid-crystal displays ${ }^{7}$. Later on, it was realized that colloidal particles, suspended in a liquid-crystal and surface-treated to impose specific orientational anchoring conditions, create defect structures that have a major role in mediating the interactions between these particles ${ }^{8-12}$. An overview of this field can be found in the comprehensive review by Kleman and Lavrentovich ${ }^{13}$. Interest in this area has remained unabated, and more recent work has covered effects such as frustration ${ }^{14}$, complex defect dynamics ${ }^{15,16}$, bistability ${ }^{17}$ and tuneable interactions between colloids ${ }^{18,19}$. However, a common element in all this work was the fact that the size of the anisotropic particles involved was irrelevant. Indeed, standard thermotropic mesogens typically have lengths below $2 \mathrm{~nm}$, and are therefore negligible in size even in micrometer-sized confining volumes or compared to the diameter of typical colloidal solutes. The physics of these systems is therefore fully captured by continuum theories ${ }^{20}$, which describe the local anisotropy of the material by vector or tensor fields that are continuous, except possibly at isolated points, lines or surfaces where defects are localized. Arguably, in some cases the nematic coherence length ${ }^{21}$, which governs the spatial extent by which boundary conditions influence the bulk behaviour, can reach dimensions comparable to the system size. However, it is an open question what happens when the size of the particles themselves does become important.

In order to reach this regime, we take a cue from nature, which provides a host of viral particles and filamentous biopolymers whose lengths are upward of the micrometer scale. Moreover, biopolymers in nature are actually often strongly confined. Cytoskeletal filaments for instance are packed at high density within thin sheet-like lamellipodia and finger-like filopodia ${ }^{22}$ and viral DNA is packed at high density inside tiny virus capsules ${ }^{23}$. These strongly confined conditions in which the polymer length is comparable to the typical dimensions of the confining space raise the general question how colloidal nematics resolve the topological and geometric constraints that inevitably arise when mutual packing and alignment to boundaries compete at the same length scale.

To address this question in a well-controlled setting, we will consider model systems composed of rod-like particles, using both simulations and experiments with $f d$-virus particles.

Since the interactions between colloidal particles, especially in physiological salt conditions, are dominated by short-range repulsions, the interactions between the particles in the simulations are taken to be of the hard body type. Since the seminal work of Onsager ${ }^{24}$, it is known that such systems display entropydriven liquid crystalline phases. The bulk phase diagram of hard rods has been studied extensively by computer simulations ${ }^{25}$. Several groups have shown that colloidal systems composed of rod-like virus particles are indeed well described by this model (for a review see ref. 26). There are few studies that have looked into the effect of confinement in a single direction ${ }^{27,28}$, but until recently studies with full confinement in all dimensions were scarce $^{29-31}$. We focus on quasi 2-dimensional (2D), circularly symmetric confinement geometries, ideally suited for analysis and visualization purposes, and readily adapted to probe different confinement topologies. In the following, we first report on the simulations, followed by the experimental results. Our main result is that, depending on the geometry and the topology of the confining volume, and the relative size and packing fraction of the particles, a wide variety of defect-mediated patterns emerge. For a circular geometry these patterns are characterized by antipodal pairs of isolated singularities, which are either located within the volume or (virtually) outside it. For the topologically distinct annular geometry, $n$-fold symmetric domain structures appear, controlled by the size of the inner hole.

\section{Results}

Circular geometry. As a canonical baseline model, we first consider a simple circular geometry. For details on the simulation technique and the simulation geometry we refer to the Methods section. As shown in Fig. 1, we observe a variety of packing structures that are all controlled by two in-plane singularities of strength $+1 / 2$. These singularities are in fact the end-points of line defects that span the system from top to bottom, but due to dominant planarization effect of the top and bottom boundaries, the system is effectively homogeneous in this direction and out of plane orientations are negligible. We therefore proceed to characterize them by their in-plane nature. A 2D disk has Euler characteristic $\chi=1$ (ref. 32), which dictates that any orientational pattern aligned to the perimeter should carry a topological charge of +1 (ref. 33). Indeed, a 2D version of continuum theory applied to the case with strong parallel boundary anchoring predicts a bipolar pattern consisting of two diametrically opposite $+1 / 2$ singularities located at the perimeter of the disc as the stable state (Supplementary Note 1; Supplementary Fig. 1). Although these simulations employ the $2 \mathrm{D}$ tensor order parameter $^{34}$, due to the observed strong degree of planarization the 3D order parameter we measure in our simulations effectively coincides with the 2D one (Supplementary Note 2). For a full discussion on our characterization of the singularities please refer to Supplementary Note 3.

Depending on the aspect ratio and the packing fraction of the particles, we find that the singularities are located inside the disc $\left(B_{\mathrm{i}}\right)$, at its boundary $\left(B_{\mathrm{b}}\right)$ or are present as virtual singularities located either a finite distance from the centre $\left(B_{\mathrm{o}}\right)$, but still causing a noticeable distortion of the alignment pattern on the inside or effectively infinitely far away $\left(B_{\infty}\right)$, yielding a nearly homogeneous nematic state. The virtually bipolar patterns $\left(B_{0}\right.$ and $\left.B_{\infty}\right)$ have been predicted to occur under weak anchoring conditions in elongated droplet shapes ${ }^{35,36}$, and reflect the fact that the entropy-driven alignment of the particles to the in-plane boundaries can at higher densities be overridden by the bulk alignment. The $B_{\mathrm{i}}$ pattern observed for shorter rods and at relatively low density in which a finite boundary layer of wall-aligned particles encloses the two singularities, however, was hitherto not described, and provides a clear signal that local packing effects can cause deviations from the continuum picture. An overview of the patterns found in the circular geometry can be found in Supplementary Table 1. Our results demonstrate that the patterns observed in strictly $2 \mathrm{D}$ systems $s^{37-39}$ are robust against the presence of a finite system size in the transverse direction ${ }^{30}$.

Annular geometry. What happens when we change the topology to that of a $2 \mathrm{D}$ annulus? This shape has Euler characteristic $\chi=0$, which suggests that singularities can be avoided. Indeed, the continuum theory of $2 \mathrm{D}$ liquid crystals with strong parallel anchoring predicts that in this case a defect-free, tangentially ordered nematic structure is stable (Supplementary Note 1; 
a

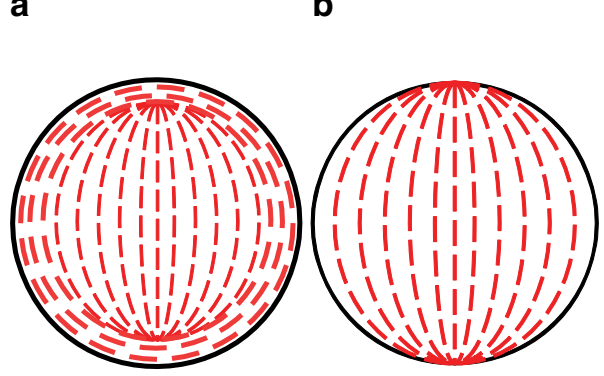

e

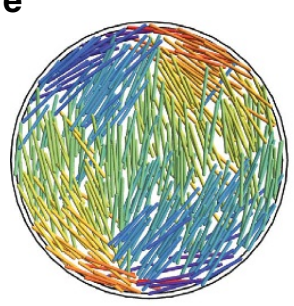

i

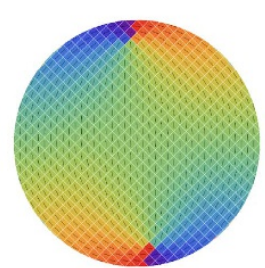

m

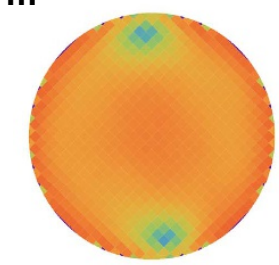

$\mathbf{q}$

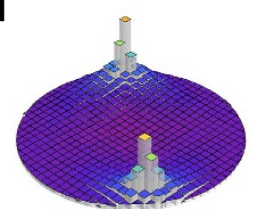

f

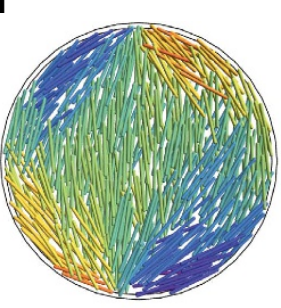

j

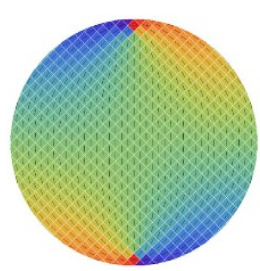

n

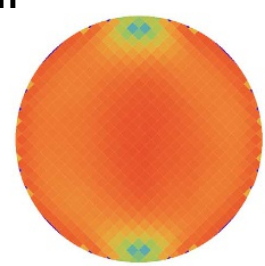

$r$

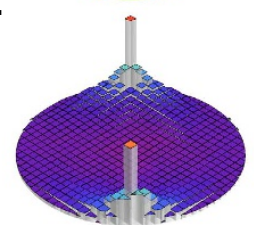

C

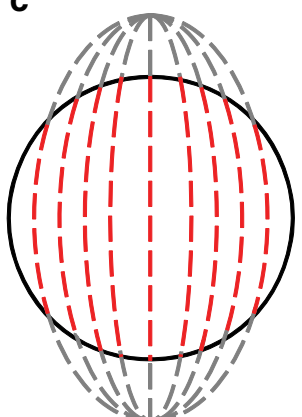

d

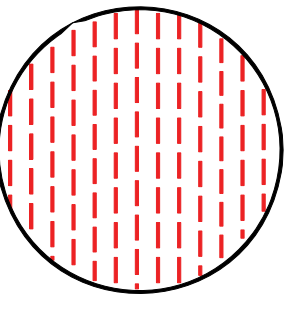

g

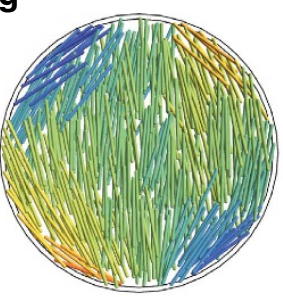

k

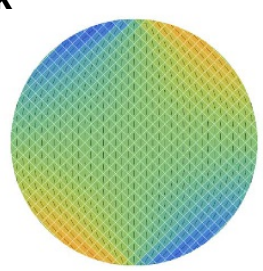

0

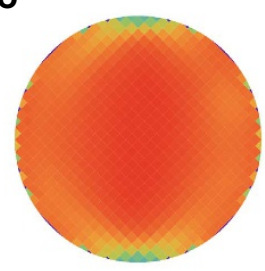

S

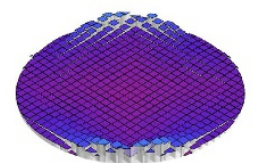

h

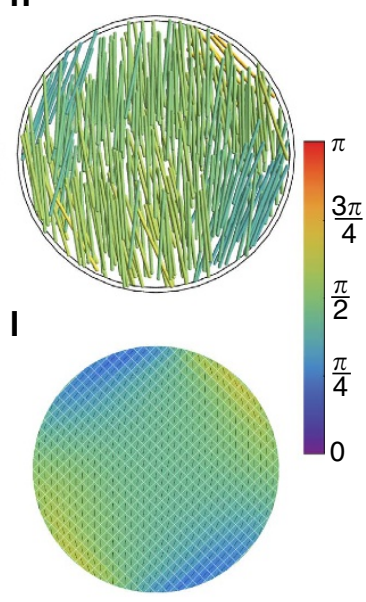

p

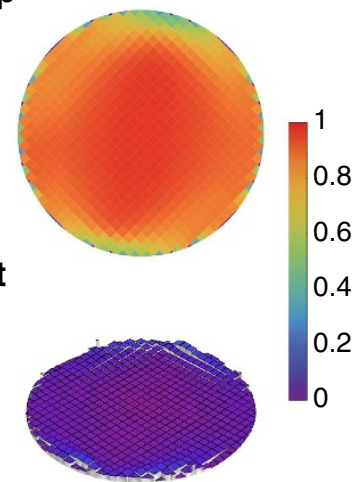

Figure 1 | Patterns of rod-like particles in circular confinement. Overview of packing structures of rod-like particles confined in shallow chambers with a circular geometry. Top row: schematic showing classification by the location of the disclination points: (a) type $B_{\mathrm{i}},(\mathbf{b})$ type $B_{\mathrm{b}}$, (c) type $B_{\mathrm{o}}$, (d) type $B_{\infty}$. Second row: snapshots showing particle positions and their orientations with respect to the vertical (mod $\pi$ ) (see colour bar on the right). Third row: orientation patterns averaged over $>10^{3}$ configurations. Fourth row: value of the scalar order parameter $S \in[0,1]$, scale bar on the right. For a discussion of the error estimate in this quantity see the Supplementary Note 8 and Supplementary Fig. 10 Note the characteristic dips at the location of the defects. Fifth row: normalized angular deficit parameter, which peaks at the centre of the defects. Simulation parameters: $(\mathbf{e}, \mathbf{i}, \mathbf{m}, \mathbf{q}) L / D=15$ and $\eta=0.16$;

$(\mathbf{f}, \mathbf{j}, \mathbf{n}, \mathbf{r}) L / D=15$ and $\eta=0.20 ;(\mathbf{g}, \mathbf{k}, \mathbf{o}, \mathbf{s}) L / D=20$ and $\eta=0.20 ;(\mathbf{h}, \mathbf{l}, \mathbf{p}, \mathbf{t}) L / D=25$ and $\eta=0.20$.

Supplementary Fig. 1). To test whether this prediction still holds for the finite particle size, we open a hole with a varying inner radius $R_{\text {inner }}$ in the centre of our simulation volume. Strikingly, we see that with increasing $R_{\text {inner, }}$ different $n$-fold symmetric domain structures develop (Fig. 2), which we denote by the dihedral group symbol $\mathrm{D}_{n}$ (ref. 40). For the case $\mathrm{D}_{3}$, we find domains with a boundary that is demarcated by $a+1 / 2$ singularity located at the outer rim and a $-1 / 2$ singularity at the inner rim. Inspection of snapshots of the simulations reveals that in this case, a particle can fit radially inside the container and form a bridge between the two singularities. For the patterns with $n \geq 4$ this is no longer possible, and the boundary between the domains is an extended disclination wall. Interestingly, a recent simulation study reported the $\mathrm{D}_{3}$ pattern as a metastable state for the $2 \mathrm{D}$ circular system ${ }^{41}$, possibly an effect of specific initial conditions. In our simulations, however, we have never observed this pattern in the circular system and we argue that it is only stable in the annular system. An overview of the patterns found in the annular geometry can be found in Supplementary Table 2.

The discrete nature of the domain structure appears to be dominated by the possible packing arrangements of the rods around a central hole of a given size. Considering the inner hole radii that would perfectly inscribe a regular $n$-gon of particles, one obtains the relation $R_{\text {inner }}(n)=\frac{1}{2 \tan (\pi / n)} L$.

Pooling all our simulation data on the observed patterns in Fig. 3, shows that indeed this geometrical criterion almost exactly 
a

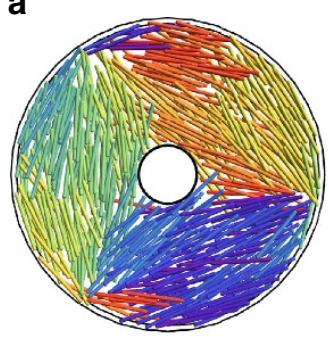

d

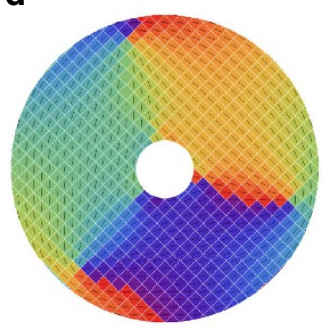

g

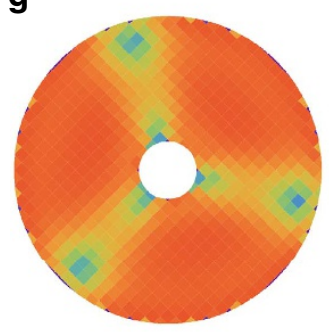

j

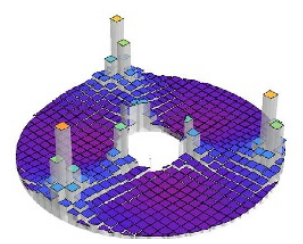

b

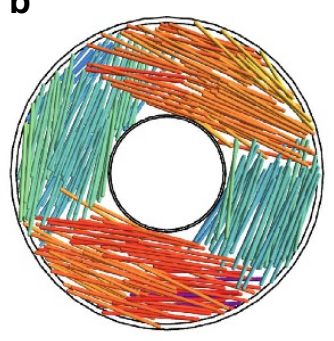

e

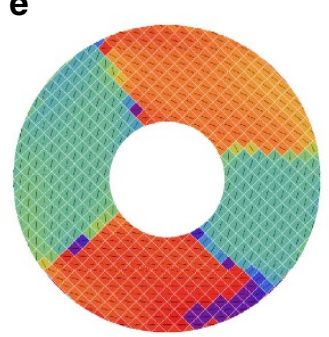

$\mathbf{h}$

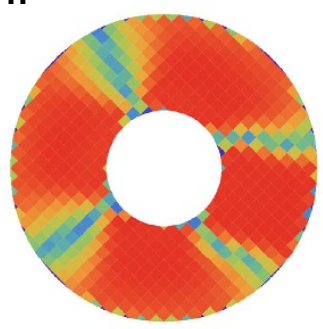

$\mathbf{k}$

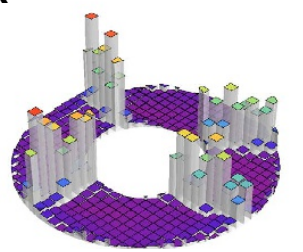

C

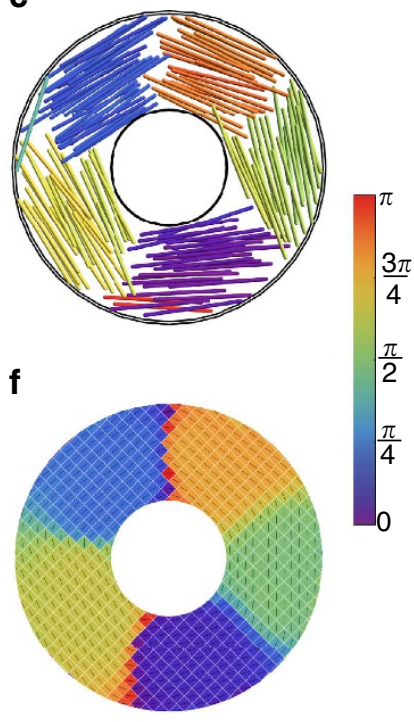

i

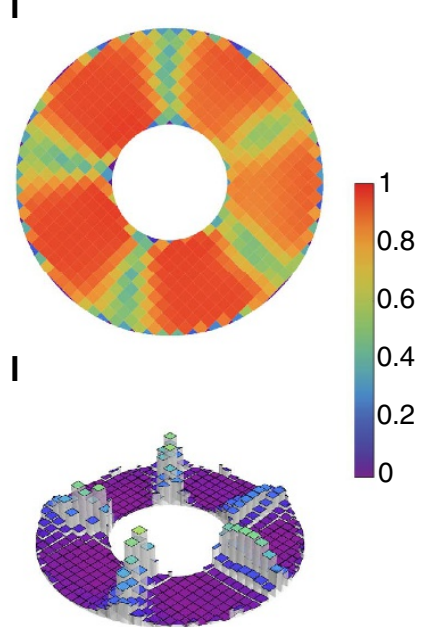

Figure 2 | Patterns of rod-like particles in annular confinement. Overview of packing structures of rod-like particles confined in annulus-shaped chambers, showing threefold (left column), fourfold (middle column) and fivefold (right column) symmetry. Top row: snapshots of simulations. Second row: particle orientations averaged over averaged over $>10^{3}$ configurations, labelled by colour bar on the right. Third row: scalar order parameter $S \in[0,1]$. Fourth row: normalized angular deficit parameter. Simulation parameters for each column (from left to right): (a,d,g,j) $H=6, L / D=15, \eta=0.20$ and $R_{\text {inner }}=7.5 ;(\mathbf{b}, \mathbf{e}, \mathbf{h}, \mathbf{k}) H=6, L / D=25, \eta=0.20$ and $R_{\text {inner }}=15 ;(\mathbf{c}, \mathbf{f}, \mathbf{i}, \mathbf{l}) H=3, L / D=25, \eta=0.20$ and $R_{\text {inner }}=15$. For error calculations see Supplementary Note 8 and Supplementary Fig. 10.

matches the observed boundaries between the different regimes for $H=D$ (Supplementary Fig. 2), while the ranges of stability of $\mathrm{D}_{3}$ and $\mathrm{D}_{4}$ patterns widen significantly for the systems with $H=3 D$ and $6 D$.

Experiments. The fact that the simulations indicate that the $\mathrm{D}_{3}$ pattern is stable for a wider range of geometries when the height of the well increases, suggests that this pattern should be observable even when the extreme transverse confinement conditions of the simulation are not fully met. We experimentally realized such a system by confining nematic liquid crystals of bacteriophage $f d$-virus $\operatorname{rods}^{42}$ inside shallow, annulus-shaped microchambers. The $f d$-virus particles are the convenient model liquid-crystal system, since they are monodisperse in length, their interactions are hard-core like when surface charges are screened and their bulk phase behaviour is well-known ${ }^{43} . f d$-rods have a length of $L=0.88 \mu \mathrm{m}$ and diameter of $D=6.6 \mathrm{~nm}$, and we can observe individual particles and their anisotropic (mostly axial) diffusion in the nematic background by fluorescence microscopy ${ }^{44}$. We use photolithography to produce nonadhesive annulus-shaped microchambers whose outer radius $R_{\text {outer }}$ ranges from 5 to $50 \mu \mathrm{m}$, yielding confinement in the range $\kappa=10^{-1}-10^{-2}$, and whose inner hole radius $R_{\text {inner }}$ ranges from 0 to 0.7 times the outer radius $R_{\text {outer }}$. The chambers have a height in the range of $H=1-3 \mu \mathrm{m}$, which is the minimal thickness that was experimentally attainable. We acquired confocal fluorescence time-lapse image series of chambers containing $f d$-rods with a small fraction of fluorescently labelled rods serving as tracers. To compute director fields, we extract the time-averaged orientation of the nematic director for each image pixel by the automated image analysis (Supplementary Note 4; Supplementary Figs 3 and 4).

We observe mainly three distinct patterns: a twofold symmetric structure $\left(D_{2}\right)$ showing two opposite $+1 / 2$ singularities (Fig. $4 \mathrm{a}$ ), a threefold symmetric pattern $\left(D_{3}\right)$ showing three $+1 / 2$ singularities (Fig. 4b) and a pattern with infinite-fold rotational symmetry $\left(D_{\infty}\right)$ that lacks singularities (Fig. $4 \mathrm{c}$ ). The occurrence 


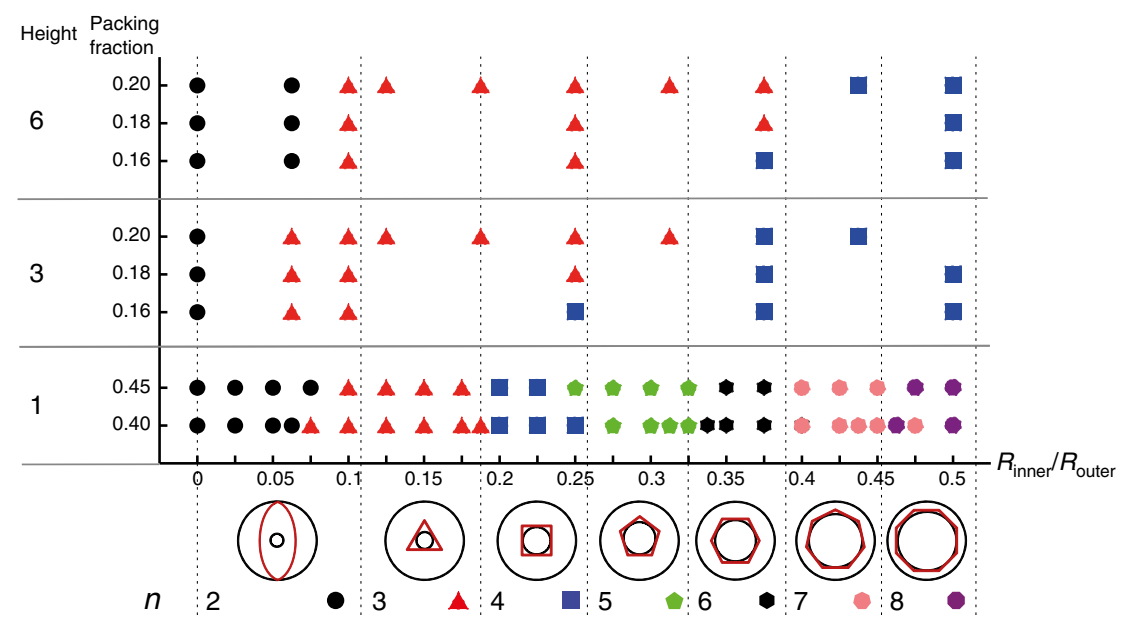

Figure 3 | Number of nematic domains in different geometries. The symmetry number $n$ of the patterns observed in simulations as a function of the inner hole radius $R_{\text {inner }}$ for rods with aspect ratio $L / D=15$ in an annular geometry with $R_{\text {outer }}=40 D$, for chamber heights $H=D$ (the strictly $2 D$ case) and $H=3 D$, $6 D$ at a number of different packing fractions. Each coloured symbol represents a different pattern, the chosen shape indicating the symmetry as explained in the legend at the bottom. The dotted vertical lines mark the predicted boundaries between the different patterns based on the geometrical rule discussed in the main text.

of these patterns strongly depends on the shape and size of the confining chambers, as shown in Fig. 4d. Strikingly, the $D_{2}$ pattern, which corresponds to the $B_{\mathrm{b}}$ pattern predicted by simulations of rods in a disk-like geometry (Fig. 1b) and in an annular geometry with small inner hole radius (Fig. 3), persists over a wide range of inner hole radii (Fig. $4 \mathrm{~d}$ ). The $D_{3}$ pattern only occurs for chambers with a small but finite hole size in the range of $1-3 \mu \mathrm{m}$, corresponding to $\sim 1-3 f d$-rod lengths and $R_{\text {inner }} / R_{\text {outer }}=0.2$ (Fig. $4 \mathrm{~d}$, red triangles). This pattern has three prominently visible and evenly spaced $+1 / 2$ singularities. Finally, the pattern with infinite-fold symmetry dominates for systems with a large inner hole $\left(R_{\text {inner }} / R_{\text {outer }}=0.7\right.$, Fig. $4 \mathrm{~d}$, blue circles $)$. The experiments are consistent with the simulation results, notably the extended stability range of the $D_{3}$ pattern in chambers of finite height. Unfortunately, we did not observe the $D_{n \geq 4}$ patterns, but note that the $D_{\infty}$ pattern is the logical limit of the patterns with increasing finite $n$-fold symmetry predicted for increasing inner hole radius. Remarkably, in spite of the fact that the experimentally realized heights in principle allow the $f d$ particles to fully rotate out of plane, the observed patterns are completely planar. This suggests that the entropically favourable planar degenerate boundary conditions imposed by the large top and bottom surfaces in conjunction with the long-ranged orientational order in the nematic state are sufficient to impose quasi-2D behaviour for a large range of box geometries. A full overview of the patterns found in the experiments is given in Supplementary Note 5 and Supplementary Figs 5 and 6.

\section{Discussion}

Our results show that strongly confined colloidal liquid crystals have a rich phase behaviour mediated by defect structures. These intricate coupled spatial and orientational patterns arise from the complex interplay between particle size and shape, and the geometry and topology of the confinement volume. The description of these effects is beyond the reach of standard continuum theories, which neglect finite particle size effects and require singularities to be incorporated 'by hand'. This raises the challenge of developing a tractable theoretical framework in which the ratio of the particle dimension to the characteristic length scale(s) is a salient parameter. However, our results show that simulations, albeit on systems that are as yet significantly smaller than the experimentally realized ones (for a critical comparison between the two please see Supplementary Note 6 and Supplementary Fig. 7), already provide predictive insights. Finally, our findings open a novel avenue to create liquid-crystal systems with designed orientational microdomain structures ${ }^{45}$, with the potential for creating controlled optical properties.

\section{Methods}

Simulations. We use the standard Metropolis Monte Carlo technique to simulate hard particles, sampling both rotations and translations of the particles, and accepting these if no overlaps with other particles or with the confining walls are created $^{46}$. As model particles, we consider hard, rigid spherocylindrical rods, of length $L$ and diameter $D$. These particles are confined in shallow, circular microchambers with radius $R_{\text {outer }}$. Two parallel plates with spacing $\mathrm{H}$ smaller than the particle length formed the top and bottom of our simulation volumes, allowing us to focus on in-plane pattern formation.

The excluded volume interactions of the rods with the walls favour planar degenerate boundary conditions ${ }^{21}$ with particles aligned parallel to the boundaries. Note, however, that, because of the finite size of the particles and the finite radius of curvature of the side walls, the centre of mass of the particles is constrained to keep a distance $>D$ from the side walls. The alignment with the lateral boundaries is therefore at best approximate. We considered both the strictly $2 \mathrm{D}$ case in which the height is equal to the diameter of the rods $(H=D)$, as well as more realistic quasi$2 \mathrm{D}$ situations, $H=3 D$ and $6 D$. We want to study the regime in which the interparticle alignment competes with the boundary alignment. Since the boundary anchoring length for spherocylindrical rods is of the order of the particle length, ${ }^{27,47}$ we need to choose $R_{\text {outer }}$ to be comparable to $L$. A convenient measure for the degree of lateral confinement is the ratio $\kappa=L /\left(2 R_{\text {outer }}\right)$, which is unity when the rods can just fit in the volume and zero for the unconfined case. In order to obtain a reasonable compromise between computational tractability and realism we used an outer radius $R_{\text {outer }}=40 D$ and particle aspect ratios in the range $L / D=15-25$, yielding $\kappa=0.2-0.3$. The number of particles was chosen to obtain packing fractions at which the corresponding unconfined system is in a nematic state $^{25}$. For the circular geometry this implies that we have between 300-500 particles for $H=6 D$. To systematically characterize any patterns observed, we measured a spatially resolved version of the standard second-rank tensorial order parameter. Local order parameter tensors have already been employed previously in the study of inhomogeneous liquid crystals, both in lattice models ${ }^{48}$ and even earlier in off-lattice models ${ }^{49}$. In these studies, the spatial variations were on a scale small with respect to the system size, but typically larger than the size of the unit cell (for the lattice models) or the particle size (in the off-lattice case). Here, however, we are using an order parameter, which is defined at a length scale smaller than that of the particles, and which also varies on this length scale. Such an order parameter has in fact already been used extensively in the study of liquid crystalline polymers (see for example, ref. 50), and has recently been shown to consistently carry over to rigid particles ${ }^{51,52}$. For more information on the order parameter, please refer to Supplementary Note 2 and Supplementary Fig. 8. The order parameter allows us to extract the average local degree of order, the local preferred axis of ordering, and the local angular deficit, which is a sensitive measure 
a
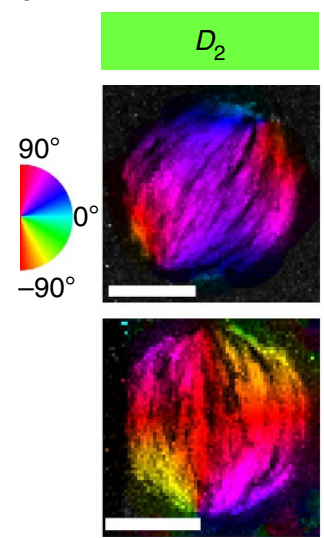

b
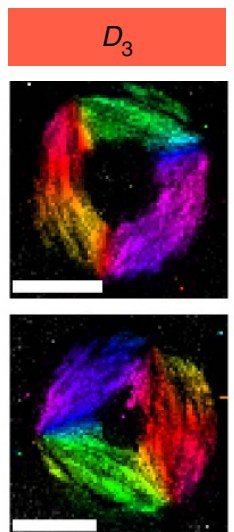

C
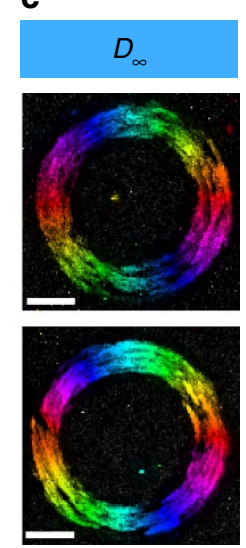

d

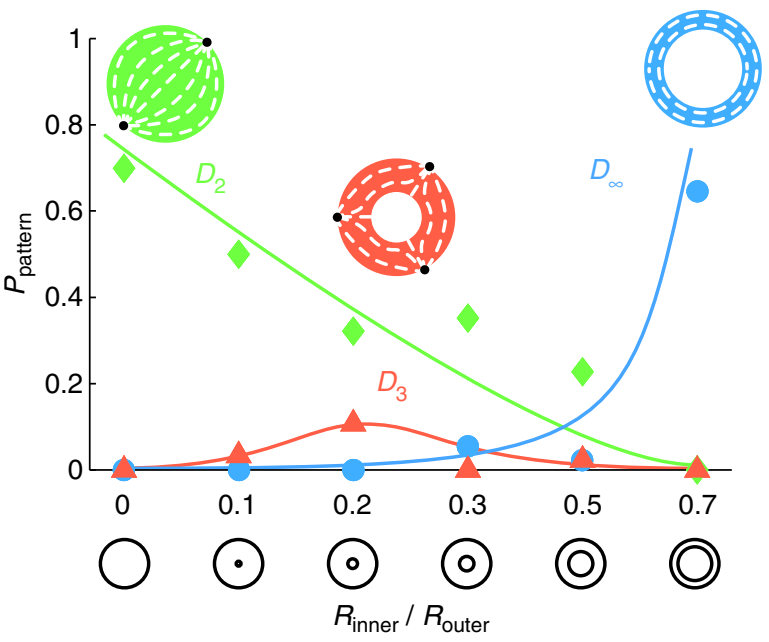

Figure 4 | Experimentally observed patterns. Structures observed in colloidal liquid crystals confined to disk-shaped and annulus-shaped microchambers. (a-c) Representative images of three types of director-field patterns. Hue corresponds to average orientation (Supplementary Note 4; Supplementary Figs 3 and 4) according to legend (left). Brightness corresponds to a maximum-time projection over 2,000 acquired frames of fluorescently labelled $f d$-virus particles. (a) $D_{2}$ : director field exhibits twofold symmetry and two singularities. (b) $D_{3}$ : director field exhibits threefold symmetry and three singularities. (c) $D_{\infty}$ : director field exhibits full rotational symmetry and no singularities. Scale bars, $5 \mu \mathrm{m}$.

(d) Probability $P_{\text {pattern }}$ that a given pattern occurs as a function of inner diameter $R_{\text {inner }}$ (in units of outer diameter $R_{\text {outer }}$ ). $D_{2}$ (green diamonds) is most likely for $R_{\text {inner }}=0 ; D_{3}$ (red triangles) is most likely for $R_{\text {inner }} / R_{\text {outer }}=0.2 ; D_{\infty}$ (blue circles) is most likely for $R_{\text {inner }} / R_{\text {outer }}=0.7$. Lines are guides to the eye. Other patterns were also found (Supplementary Note 5; Supplementary Figs 5 and 6).

for the presence of orientational singularities (Supplementary Note 7). It should be noted that these singularities are in the orientation field, and are not accompanied by any significant change in the local packing fraction of the rods (Supplementary Fig. 9). To monitor the equilibration of our system, we use the largest positive eigenvalue of the global tensor order parameter as a scalar measure of ordering. To analyze the statistical reliability of our results we have performed an error analysis using subsamples, which is described in Supplementary Note 8 and Supplementary Fig. 10.

Bacteriophage $\boldsymbol{f d}$-virus preparation. $f d$-virus rods were grown using a standard protocol $^{26}$ and stored in $f d$-buffer solution $(20 \mathrm{mM}$ tris, $\mathrm{pH} 8.15,100 \mathrm{mM}$ sodium chloride, $15 \%$ ethanol). Assay suspensions were prepared at concentrations of $24 \mathrm{mg} \mathrm{ml}^{-1}$, slightly above the bulk isotropic-nematic biphasic region, which occurs at $\sim 20 \mathrm{mg} \mathrm{ml}^{-1}$, in agreement with the Onsager theory ${ }^{53}$. Bulk suspensions were biphasic, as evidenced by visual inspection through crossed polarizers. We chose the lowest possible nematic concentration, in order to minimize the energy cost to re-arrange from one director field to another and thus reduce the probability of getting stuck in high-energy metastable states. Fluorescently labelled rods were prepared by incubation with Alexa-488 succinimidyl ester (Invitrogen) following a published procedure ${ }^{47}$. A small fraction of labelled rods $(2-4 \% \mathrm{v} / \mathrm{v})$ was mixed with unlabelled rods in order to make individual-labelled rods distinguishable by fluorescence microscopy.

Microchamber preparation. Microchambers were assembled using a standard photolithographic technique described elsewhere ${ }^{30}$. Chamber dimensions were set by a mask design with circular and annular geometries with outer radii of $5,10,15$, 25,35 and $50 \mu \mathrm{m}$. For each outer radius, geometries with inner radii of $0,0.1,0.2$, $0.3,0.5$ and 0.7 times the outer radius were made, resulting in a total of 36 different geometries. Chambers were sealed with polydimethylsiloxane-coated microscope slides and soaked overnight in $f d$-buffer containing $0.1 \mathrm{wt} \%$ of the amphiphilic block copolymer Pluronic F-127 (Sigma-Aldrich). This treatment effectively blocked nonspecific adsorption of $f d$-rods, as confirmed by time-lapse imaging of rod diffusion. Saturation of the PDMS with buffer prevented drying of the sample for at least $24 \mathrm{~h}$.

Confinement assay. A drop of $f d$-virus suspensions was placed on a glass-photoresist substrate and pressed against rubber-coated glass to form microchambers. We hermetically sealed the glass edges with VALAP and let samples equilibrate for at least $30 \mathrm{~min}$ before visualizing by fluorescence microscopy. We only consider well-sealed chambers for quantification. Approximately $40 \%$ of all chambers are well-sealed. Chambers that were not well-sealed were evident by fluorescently labelled rods escaping from the chamber. The rods rapidly organized in steadystate nematic patterns within $30 \mathrm{~min}$ after filling the chambers. When we filled the chambers with the isotropic phase below the biphasic region, a nematic was not formed and the samples remained isotropic. This observation implies that the filling (and confinement) were not sufficient to induce a nematic.

Fluorescence microscopy. Microchambers were visualized using two microscope setups: (1) a spinning disk confocal scanner (CSU 22, Yokogawa) on an inverted microscope (DMIRB, Leica) with a cooled, electron-multiplying charged-coupled device (C9100, Hamamatsu) and (2) a Nikon C1 confocal point scanner on an inverted microscope (Ti, Nikon) with a photomultiplier tube detector (A1, Nikon). Labelled rods were excited with $488 \mathrm{~nm}$ laser light (Coherent). A series of images were recorded over a long enough time interval such that viruses diffused across the entire chamber. The average diffusion constant of virus rods in the nematic phase is $1 \mu \mathrm{m}^{2} \mathrm{~s}^{-1}$ along the nematic director and $0.1 \mu \mathrm{m}^{2} \mathrm{~s}^{-1}$ perpendicular to the nematic director ${ }^{44}$. These diffusion constants result in diffusion timescales of tens of seconds for diffusion over a distance of one particle length and minutes for diffusing over interparticle distances between fluorescently labelled rods $(\sim$ a few $\mu \mathrm{m})$. For spinning disk data, typically ten movies of 200 frames each were acquired at a fast imaging rate ( 0.1 frames per second), which were separated by $2 \mathrm{~min}$ to allow rods to diffuse completely across the chamber. For point-scanning confocal data, $15-30$ frames were acquired at a slower rate (1 frame per $\sim 1-2 \mathrm{~min}$ ) over several fields of view which were automatically acquired and stitched (NIS Elements, Nikon). A customized image analysis technique was developed to determine the average nematic director orientation $\langle\theta\rangle$ given time-averaged orientations $\theta$ of labelled $f d$-rods across all images acquired (Supplementary Note 4; Supplementary Figs 3 and 4 ).

Identification of nematic patterns. Rods formed a variety of liquid-crystal patterns. The pattern type was determined by visual inspection of the nematic director fields of all well-filled chambers on the chip. Supplementary Figs 11 and 12 summarize the pattern frequency and probability, respectively, as a function of $R_{\text {outer }}$ and $R_{\text {inner }} / R_{\text {outer }}$. We define the probability that a pattern $P$ occurs in a given chamber geometry $G$ as the number of observed instances of $P$ divided by the total number of well-sealed chambers with geometry $G$. A total of 243 chambers were analyzed: $80,27,26,36,43$ and 31 chambers for $R_{\text {inner }} / R_{\text {outer }}=0,0.1,0.2,0.3,0.5$ and 0.7 , respectively; $77,75,55,25,9$ and 2 chambers for $R_{\text {outer }} / \mu \mathrm{m}=5,10,15,25$, 35 and 50, respectively; $113,5,23,21,33,32$ and 16 chambers for patterns $D_{2}, D_{3}$, $D_{\infty}, \mathrm{N}, \mathrm{A}_{1}, \mathrm{~A}_{2}$, and $\mathrm{A}_{+}$, respectively.

Data availability. The data that support the findings of this study, as well as the computer codes used in the simulations, are available from the corresponding author upon request.

\section{References}

1. Castellano, J. A. Liquid Gold: The Story Of Liquid Crystal Displays and the Creation of an Industry (World Scientific Publishing Company, 2005).

2. Kléman, M., Michel, L. \& Toulouse, G. Classification of topologically stable defects in ordered media. J. Phys. Lett. 38, 195-197 (1977).

3. Kléman, M. \& Michel, L. On the classification of defects in the smectic phases Sm A and Sm C. J. Phys. Lett. 39, 29-32 (1978). 
4. Volovik, G. E. \& Mineev, V. P. Investigation of singularities in superfluid $\mathrm{He} 3$ in liquid crystals by the homotopic topology methods. J. Exp. Theor. Phys. 72, 2256-2274 (1977).

5. Volovik, G. E. \& Lavrentovich, O. D. Topological dynamics of defects: boojums in nematic drops. J. Exp. Theor. Phys. 58, 1159 (1983).

6. Lavrentovich, O. D. \& Terent'ev, E. M. Phase transition altering the symmetry of topological point defects (hedgehogs) in a nematic liquid crystal. J. Exp. Theor. Phys. 64, 1237 (1986).

7. Doane, J. W., Golemme, A., West, J. L., Whitehead, J. B. \& Wu, B.-G. Polymer dispersed liquid crystals for display application. Mol. Cryst. Liq. Cryst. Inc. Nonlinear Opt. 165, 511-532 (1988).

8. Kuksenok, O. V., Ruhwandl, R. W., Shiyanovskii, S. V. \& Terentjev, E. M. Director structure around a colloid particle suspended in a nematic liquid crystal. Phys. Rev. E 54, 5198-5203 (1996).

9. Ruhwandl, R. W. \& Terentjev, E. M. Monte Carlo simulation of topological defects in the nematic liquid crystal matrix around a spherical colloid particle. Phys. Rev. E 56, 5561-5565 (1997).

10. Ruhwandl, R. W. \& Terentjev, E. M. Long-range forces and aggregation of colloid particles in a nematic liquid crystal. Phys. Rev. E 55, 2958-2961 (1997).

11. Poulin, P. Novel colloidal interactions in anisotropic fluids. Science 275, 1770-1773 (1997).

12. Poulin, P., Francès, N. \& Mondain-Monval, O. Suspension of spherical particles in nematic solutions of disks and rods. Phys. Rev. E 59, 4384-4387 (1999).

13. Kleman, M. \& Lavrentovich, O. D. Topological point defects in nematic liquid crystals. Philos. Mag. 86, 4117-4137 (2006).

14. Lopez-Leon, T., Koning, V., Devaiah, K. B. S., Vitelli, V. \& Fernandez-Nieves, A. Frustrated nematic order in spherical geometries. Nat. Phys. 7, 391-394 (2011).

15. Lopez-Leon, T., Bates, M. A. \& Fernandez-Nieves, A. Defect coalescence in spherical nematic shells. Phys. Rev. E 86, 030702 (2012).

16. Tasinkevych, M., Campbell, M. G. \& Smalyukh, I. I. Splitting, linking, knotting, and solitonic escape of topological defects in nematic drops with handles. Proc. Natl Acad. Sci. USA 111, 16268-16273 (2014).

17. Tsakonas, C., Davidson, A. J., Brown, C. V. \& Mottram, N. J. Multistable alignment states in nematic liquid crystal filled wells. Appl. Phys. Lett. 90, 111913 (2007)

18. Lapointe, C. P., Mason, T. G. \& Smalyukh, I. I. Shape-controlled colloidal interactions in nematic liquid crystals. Science 326, 1083-1086 (2009)

19. Senyuk, B. et al. Topological colloids. Nature 493, 200-205 (2013).

20. Campbell, M. G., Tasinkevych, M. \& Smalyukh, I. I. Topological polymer dispersed liquid crystals with bulk nematic defect lines pinned to handlebody surfaces. Phys. Rev. Lett. 112, 197801 (2014).

21. de Gennes, P. G. \& Prost, J. The Physics of Liquid Crystals (International Series of Monographs on Physics) (Oxford University Press, 1995).

22. Medalia, O. et al. Macromolecular architecture in eukaryotic cells visualized by cryoelectron tomography. Science 298, 1209-1213 (2002).

23. Speir, J. A. \& Johnson, J. E. Nucleic acid packaging in viruses. Curr. Opin. Struct. Biol. 22, 65-71 (2012).

24. Onsager, L. The effects of shape on the interaction of colloidal particles. Ann. N. Y. Acad. Sci. 51, 627-659 (1949).

25. Bolhuis, P. \& Frenkel, D. Tracing the phase boundaries of hard spherocylinders. J. Chem. Phys. 106, 666 (1997).

26. Dogic, Z. \& Fraden, S. in Soft Matter, Vol. 2: Complex Colloidal Suspensions (eds Gompper, G. \& Schick, M.) 1-86 (Wiley-VCH Verlag GmbH \& Co. KGaA, 2006).

27. Dijkstra, M., Roij, R. \& Evans, R. Wetting and capillary nematization of a hardrod fluid: a simulation study. Phys. Rev. E 63, 051703 (2001).

28. Cosentino Lagomarsino, M., Dogterom, M. \& Dijkstra, M. Isotropic-nematic transition of long, thin, hard spherocylinders confined in a quasi-twodimensional planar geometry. J. Chem. Phys. 119, 3535 (2003).

29. Trukhina, Y. \& Schilling, T. Computer simulation study of a liquid crystal confined to a spherical cavity. Phys. Rev. E 77, 011701 (2008).

30. Lewis, A. H. et al. Colloidal liquid crystals in rectangular confinement: theory and experiment. Soft Matter 10, 7865-7873 (2014).

31. Gârlea, I. C. \& Mulder, B. M. Defect structures mediate the isotropic-nematic transition in strongly confined liquid crystals. Soft Matter 11, 608-614 (2015).

32. Kinsey, L. C. Topology of Surfaces (Springer Science \& Business Media, 1993).

33. Alexander, G. P., Chen, B. G., Matsumoto, E. A. \& Kamien, R. D. Colloquium: disclination loops, point defects, and all that in nematic liquid crystals. Rev. Mod. Phys. 84, 497-514 (2012).

34. Barci, D. G. \& Stariolo, D. A. Orientational order in two dimensions from competing interactions at different scales. Phys. Rev. B 79, 075437 (2009).

35. Prinsen, P. \& van der Schoot, P. Shape and director-field transformation of tactoids. Phys. Rev. E 68, 021701 (2003).

36. Prinsen, P. \& van der Schoot, P. Continuous director-field transformation of nematic tactoids. Eur. Phys. J. E. Soft Matter 13, 35-41 (2004).
37. Ondris-Crawford, R. J., Crawford, G. P., Zumer, S. \& Doane, J. W. Curvatureinduced configuration transition in confined nematic liquid crystals. Phys. Rev. Lett. 70, 194-197 (1993).

38. Jian, K., Hurt, R. H., Sheldon, B. W. \& Crawford, G. P. Visualization of liquid crystal director fields within carbon nanotube cavities. Appl. Phys. Lett. 88, 163110 (2006).

39. de lasHeras, D., Velasco, E. \& Mederos, L. Topological defects in a twodimensional liquid crystal confined in a circular nanocavity. Phys. Rev. E 79, 061703 (2009)

40. Jacobson, N. Basic Algebra I (Dover Books, 2009).

41. de las Heras, D. \& Velasco, E. Domain walls in two-dimensional nematics confined in a small circular cavity. Soft Matter 10, 1758-1766 (2014).

42. Berkowitz, S. A. \& Day, L. A. Mass, length, composition and structure of the filamentous bacterial virus fd. J. Mol. Biol. 102, 531-547 (1976).

43. Dogic, Z. \& Fraden, S. Ordered phases of filamentous viruses. Curr. Opin. Colloid Interface Sci. 11, 47-55 (2006).

44. Lettinga, M. P. \& Grelet, E. Self-diffusion of rodlike viruses through smectic layers. Phys. Rev. Lett. 99, 197802 (2007)

45. Mendonca, C. R. et al. Three-dimensional fabrication of optically active microstructures containing an electroluminescent polymer. Appl. Phys. Lett. 95, 113309 (2009)

46. Frenkel, D. \& Smit, B. Understanding Molecular Simulation, Second Edition: From Algorithms to Applications (Computational Science) (Academic Press, 2001).

47. Dammone, O. et al. Confinement induced splay-to-bend transition of colloidal rods. Phys. Rev. Lett. 109, 108303 (2012).

48. Chiccoli, C., Pasini, P., Sarlah, A., Zannoni, C. \& Zumer, S. Structures and transitions in thin hybrid nematic films: a Monte Carlo study. Phys. Rev. E. Stat. Nonlinear Soft Matter Phys. 67, 050703 (2003).

49. Allen, M. P., Warren, M. A., Wilson, M. R., Sauron, A. \& Smith, W. Molecular dynamics calculation of elastic constants in Gay-Berne nematic liquid crystals. J. Chem. Phys. 105, 2850 (1996).

50. Khokhlov, A. R. \& Grosberg, A. Y. Statistical Physics of Macromolecules (AIP, 1994).

51. Jiang, Y. \& Chen, J. Z. Y. Isotropic - nematic interface in a lyotropic system of wormlike chains with the Onsager interaction. Macromolecules 43, 10668-10678 (2010).

52. Chen, J. Z. Y. Structure of two-dimensional rods confined by a line boundary. Soft Matter 9, 10921 (2013).

53. Tang, J. \& Fraden, S. Isotropic-cholesteric phase transition in colloidal suspensions of filamentous bacteriophage fd. Liq. Cryst. 19, 459-467 (1995).

\section{Acknowledgements}

This work is part of the research programme of the Foundation for Fundamental Research on Matter (FOM), which is part of the Netherlands Organisation for Scientific Research (NWO). G.H.K. also acknowledges a Vidi grant from the Netherlands Organization for Scientific Research (NWO).

\section{Author contributions}

I.C.G., D.G.A.L.A., M.P.L., G.H.K. and B.M.M. conceived and designed the simulations and the experiments. I.C.G., P.M., J.A. and O.D. performed the simulations and the experiments and analyzed the data. I.C.G., J.A., D.G.A.L.A., M.P.L. and B.M.M. contributed materials and analysis tools. I.C.G., J.A., D.G.A.L.A., M.P.L. and B.M.M. wrote the paper.

\section{Additional information}

Supplementary Information accompanies this paper at http://www.nature.com/ naturecommunications

Competing financial interests: The authors declare no competing financial interests

Reprints and permission information is available online at http://npg.nature.com/ reprintsandpermissions/

How to cite this article: Gârlea, L. C. et al. Finite particle size drives defect-mediated domain structures in strongly confined colloidal liquid crystals. Nat. Commun. 7:12112 doi: $10.1038 /$ ncomms12112 (2016).

\begin{abstract}
This work is licensed under a Creative Commons Attribution 4.0 International License. The images or other third party material in this article are included in the article's Creative Commons license, unless indicated otherwise in the credit line; if the material is not included under the Creative Commons license, users will need to obtain permission from the license holder to reproduce the material. To view a copy of this license, visit http://creativecommons.org/licenses/by/4.0/
\end{abstract}

(C) The Author(s) 2016 


\title{
Erratum: Finite particle size drives defect-mediated domain structures in strongly confined colloidal liquid crystals
}

\author{
loana C. Gârlea, Pieter Mulder, José Alvarado, Oliver Dammone, Dirk G.A.L. Aarts, M. Pavlik Lettinga, \\ Gijsje H. Koenderink \& Bela M. Mulder
}

Nature Communications 7:12112 doi: 10.1038/ncomms12112 (2016); Published 29 Jun 2016; Updated 3 Aug 2016

The present address for Ioana C. Gârlea is incorrect in this Article. The correct present address for this author is given below:

Faculty of Physics, University of Vienna, Boltzmanngasse 5, A-1090 Vienna, Austria.

The name of this author is also incorrect in the 'How to cite this article' section. This section should read:

Gârlea, I. C. et al. Finite particle size drives defect-mediated domain structures in strongly confined colloidal liquid crystals. Nat. Commun. 7:12112 doi: 10.1038/ncomms12112 (2016).

(c) This work is licensed under a Creative Commons Attribution 4.0 International License. The images or other third party material in this article are included in the article's Creative Commons license, unless indicated otherwise in the credit line; if the material is not included under the Creative Commons license, users will need to obtain permission from the license holder to reproduce the material. To view a copy of this license, visit http://creativecommons.org/licenses/by/4.0/ 